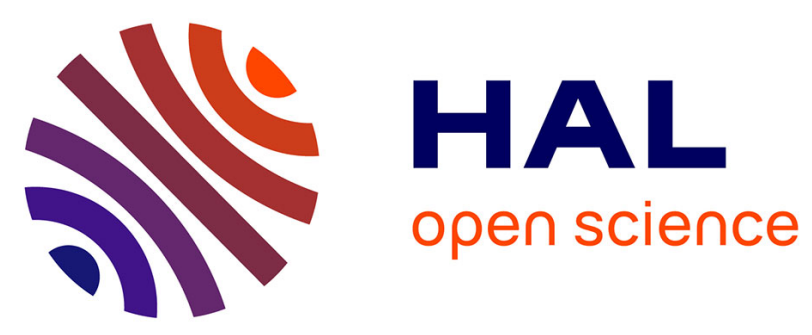

\title{
Régions et télécommunication en Europe du Sud
}

Emmanuel Négrier

\section{To cite this version:}

Emmanuel Négrier. Régions et télécommunication en Europe du Sud. NETCOM : Réseaux, communication et territoires / Networks and Communications Studies, 1995, 8 (2), pp.426 - 445. 10.3406/netco.1994.1236 . hal-02515810

\section{HAL Id: hal-02515810 \\ https://hal.science/hal-02515810}

Submitted on 24 Apr 2020

HAL is a multi-disciplinary open access archive for the deposit and dissemination of scientific research documents, whether they are published or not. The documents may come from teaching and research institutions in France or abroad, or from public or private research centers.
L'archive ouverte pluridisciplinaire HAL, est destinée au dépôt et à la diffusion de documents scientifiques de niveau recherche, publiés ou non, émanant des établissements d'enseignement et de recherche français ou étrangers, des laboratoires publics ou privés.

\section{(이) $\$$}

Distributed under a Creative Commons Attribution - NonCommercial - NoDerivatives| 4.0 
NETCOM : Réseaux, communication et territoires /

\section{Régions et télécommunications en Europe du Sud}

\section{Emmanuel Négrier}

\section{Citer ce document / Cite this document :}

Négrier Emmanuel. Régions et télécommunications en Europe du Sud. In: NETCOM : Réseaux, communication et territoires / Networks and Communication Studies, vol. 8 n², octobre 1994. pp. 426-445;

doi : https://doi.org/10.3406/netco.1994.1236

https://www.persee.fr/doc/netco_0987-6014_1994_num_8_2_1236

Fichier pdf généré le 11/02/2020 
SYMPOSIUM: "TELECOMMUNICATIONS, GEOGRAPHIE POLITIQUE ET CHANGEMENT GLOBAL"

Union Géographique Internationale

Union Internationale de Sciences Politiques

Paris/Issy-les-Moulineaux

10-13 mai 1993

\section{RÉGIONS ET TÉLÉCOMMUNICATION EN EUROPE DU SUD}

présenté par Emmanuel NEGRIER

Chercheur CNRS, CEPEL-Montpellier $\mathrm{I}^{1}$

\section{Introduction:}

Les rapports entre télécommunication et régions n'appartiennent pas, à quelques rares exceptions près, à l'histoire territoriale du secteur ${ }^{2}$. Pour des motifs politiques, technologiques et économiques, le déploiement en Europe d'une conception publique des télécoms a coïncidé avec une acception nationale de sa mise en oeuvre. La fin des années 1980 a inauguré une large mise en question de l'ensemble de ces justifications: développement, à partir du Livre Vert de 1987, d'une échelle européenne de la réglementation et de la recherche-développement; différenciation des attentes qu'une conception de "service public" entendait traiter de façon homogène; mise en question des modèles socio-politiques d'encadrement du secteur...

L'hypothèse peut être faite que cet ensemble de mouvements, le plus souvent traités par le haut, ont également une signification territoriale et politique. Contemporaine de cette évolution globale, le développement, en Europe du Sud, de dispositifs de décentralisation dans des pays de "tradition napoléonienne", a en particulier reposé la question de l'inertie politique des institutions locales dans ce secteur, quand elle ne leur a pas donné directement l'occasion de s'y intéresser (cas du câble en France, mais aussi, en Italie, à travers la récente réforme de la réglementation). Deux autres spécificités de l'Europe du Sud, à laquelle

\footnotetext{
1 Ce texte représente une partie d'un travail collectif mené par une équipe du CEPEL constituée, outre I'auteur, d'Alain BRIOLE, Richard LAURAIRE et Luis De La TORRE.. Le rapport, pour le Commissariat Général du Plan, s'intitule: "les politiques publiques de télécommunication en Europe du Sud. Service public et dynamiques territoriales des intérêts". Il est disponible auprès du CEPEL, 39, rue de l'Université, 34060 Montpellier Cedex

${ }^{2}$ Cf. E.NEGRIER, L.DE LA TORRE: "Régions et télécommunications en Europe du Sud" Actes du Congrès de la SFIC, Inforcom, Lille, Mai 1992
} 
appartiennent ces pays, concernent directement le secteur des télécoms: une situation relative de moindre développement, qui implique l'absence, en particulier, d'une logique intégrée du "champion national", et les rend plus sensibles aux logiques de déploiement en Europe des multinationales europénnes, mais surtout extra-européennes, comme ATT par exemple; un mode d'encadrement institutionnel et politique des télécoms assez atypique: pluralité d'opérateurs publics de statuts distincts en Italie, concession à un opérateur où l'Etat n'est qu'un actionnaire minoritaire, en Espagne.

C'est le croisement de ces évolutions sectorielles et d'un mode de développement régional des attentes de service dont nous allons présenter les conditions d'émergence, les principales manifestations et les enjeux, à travers la référence aux Régions d'Emilie-Romagne, de Lombardie, de Catalogne et de Madrid (Communauté autonome), ainsi qu' à celles du Sud de la France. Les investigations de terrain sur cet ensemble de sites permettent de dégager d'utiles enseignements comparés sur la genèse de l'intérêt régional pour ce secteur ainsi que sur la production intellectuelle de positions régionales. Elle permettent en outre de montrer que l'évolution du service public, souvent référé à des mouvements de déstabilisation de niveau national et international, est bien aussi celle de son organisation territoriale. Enfin, la dimension européenne, assez récemment apparue dans ce secteur, peut apparaître comme un des nouveaux enjeux d'un service public envisagé à partir du niveau régional.

\section{TÉLÉCOMMUNICATION ET INTÉRÊT RÉGIONAL}

Quels sont les facteurs d'investissement des collectivités et acteurs localisés dans les télécommunications ? Eu égard aux contraintes héritées de l'histoire, dans chacun des pays et territoires régionaux, on peut dresser un tableau assez comparable des motivations et processus à travers lesquels cette évolution se fait jour.

\section{Télécommunication et développement économique régional}

Tout d'abord, le pôle principal paraît être, dans chacun des sites étudiés, la notion de développement économique. Cette dimension, 
très présente dans les discours officiels qui inaugurent les initiatives publiques locales, est tout à la fois liée à un expérience préalable de politiques économiques régionales et aux professionnels locaux de ce domaine.

Les politiques économiques régionalisées liées à un contexte de décentralisation politico-administrative croisé avec une théorisation, datant des années 1980, de la décentralisation économique. Cette rupture consommée avec, d'une part, la centralisation étatique des ressources et des modes d'allocation liées au développement, et d'autre part, face au modèle de concentration des firmes, émerge une nouvelle structure de relations, régionalisée, entre autorités publiques locales et petites et moyennes entreprises.

C'est sur cette nouvelle structure que se greffe, de façon dominante, les croisements entre développement économique et télécommunications. Pour deux raisons essentielles, qui tiennent d'une part à la situation de cette catégorie d'entreprises (et singulièrement les PME de l'industrie des services, dont l'activité repose sur une utilisation optimale des nouvelles technologies de communication), mais aussi au rôle original que les pouvoirs régionaux entendent jouer (ou que l'on entend qu'ils jouent) dans ce secteur.

La situation des PME vis à vis du secteur des télécommunications est généralement marquée, en Espagne, comme en France et en Italie, par un double déficit. Les niveaux d'investissement requis pour l'équipement en infrastructures et services de pointe demeurent hors de portée d'une unité singulière. L'expertise nécessaire à leur utilisation et à leur valorisation échappe, encore largement monopolisée par des corps de professionnels particuliers, étrangers la plupart du temps au milieu des PME. Cette situation peut être considérée comme un des éléments objectifs de ce que certains ont qualifié de "demande de région", comme elle fait l'objet d'une démarche particulière de la part des autorités européennes, notamment dans le cadre des programmes STAR et TELEMATIQUE. Les autorités régionales se sont mobilisées sur un tel champ comme des instances susceptibles d'offrir des prestations que ni l'opérateur public, ni les firmes elles-mêmes n'étaient toujours en mesure d'offrir dans des conditions satisfaisantes. La participation des collectivités locales à l'implantation de zones de télécommunication, au développement de services intègrés une dimension de "socialisation" des équipements et réseaux auprès d'un public particulier. 
A ce stade, les structures économiques locales jouent un rôle non-négligeable dans le croisement, plus ou moins intense et théorisé, entre télécommunication et intérêts économiques régionaux. L'Emilie-Romagne et la Lombardie constituent respectivement deux modèles opposés d'investissement politique local. Le premier cas est bien connu pour être l'illustration d'une économie diffuse de petites unités économiques. La politisation des télécommunications s'y fait sur un mode radicalement distinct de la Lombardie. Elle combine trois traits principaux: une élaboration associée à une dimension plus large de planification économique territoriale; le rejet d'une forme de concentration des équipements et réseaux par pôle dominant; un dispersion de la mise en oeuvre entre assessorats sectoriels, plus tournés vers les applications et services que vers les infrastructures elles-mêmes.

Dans le cas lombard à l'inverse, la présence d'intérêts économiques polarisés dans la métropole milanaise, par ailleurs forts consommateurs de télécommunication et liés mêmes aux intérêts nationaux et internationaux du secteur, a entraîné une réflexion d'une toute autre nature. L'aire lombarde a été très tôt considérée comme pouvant représenter une territoire de compétition (les années 1980 étant celles des grandes craintes quant à la dérégulation brutale du secteur) mais aussi d'expérimentation. C'est ce qui explique le jeu ambigü de l'opérateur public sur le projet "Lombardia cablata": une présence active dans les orientations et un freinage de toute initiative concrète. C'est aussi ce qui permet de comprendre une démarche axée sur les infrastructures.

On retrouve cette même importance des structures économiques régionales dans les cas français et espagnols. Les démarches madrilène et catalane, comme en Midi-Pyrénées ou en Languedoc Roussillon, font une place importante à la petite entreprise innovante (que ce soit dans les projets de téléport, de ZTA, ou de services à valeur ajoutée), la plus directement concernée par les initiatives publiques régionales.

Le poids du facteur directement économique dans l'investissement des pouvoirs régionaux est cependant relatif. D'une part, il est lié à une représentation du territoire en termes de compétition, nationale et européenne. De manière assez paradoxale, la genèse des projets d'intervention est associée à un discours tenu sur la corrélation entre le développement économique et la disposition locale d'infrastructures et de services de télécommunications, alors même que la plupart des recherches concluent à la grande relativité 
des effets de ceux-ci sur la localisation des activités. C'est ce qui explique la récurrence des études commandées par les institutions régionales sur l'état comparé des prestations, non seulement aux niveaux intra et inter-régional, mais aussi à l'échelle européenne. Le choix de cette dernière n'est pas neutre, notamment en Italie et en Espagne.

Pour des régions fortement développées comme la Catalogne et la Lombardie, ce niveau de comparaison est directement lié à une mise en question de l'Etat comme sphère pertinente d'allocation et de régulation du secteur. Cette mise en cause revêt deux dimensions opposées: en Lombardie, une représentation de l'Etat italien en termes d'inefficacité; en Catalogne, la volonté de produire et reproduire un particularisme à l'égard d'une régulation centrale "castillane". Dans les deux cas, la volonté de situer les "véritables enjeux" au niveau européen est une façon d'échapper aux contraintes de régulation nationale (et notamment la péréquation territoriale).

Cette rhétorique de la compétition est ensuite liée à la question de la légitimité intra-régionale, entre exécutifs et territoires de référence. Dans la plupart des cas, le lancement d'initiatives régionales est en relation directe avec une discussion sur le niveau adéquat de régulation sub-étatique. Qu'il s'agisse de la Catalogne, entre la Ville de Barcelone et la Généralité, de la Lombardie, entre la Ville de Milan et la Région lombarde, ou encore du LanguedocRoussillon, entre la Ville de Montpellier et le Conseil Régional, la rivalité des Exécutifs constitue, dans ce domaine comme dans d'autres, un facteur de déclanchement important. Les politiques des niveaux régionaux sont antérieurs aux initiatives des villes-centre dans les cas lombard et émilien, alors qu'elles leur succèdent dans les cas languedocien et catalan.

Cette lutte pour la légitimité sectorielle et territoriale n'est pas simplement liée à une course à l'anticipation. Elle intervient aussi dans le cadre d'une compétition plus large sur le terrain du croisement autorités publique régionales/intérêts économiques localisés. L'affichage de prétentions publiques dans un secteur à forte innovation d'infrastructure et de services est lié à la question du leadership territorial sur la programmation économique et la représentation des intérêts qu'elle comporte. En ce sens, la projection d'infrastructures purement urbaines ou régionalisées est contemporaine des efforts des mêmes exécutifs pour doter leur niveau territorial de structures sociales adéquates dans le domaine 


\section{1}

économique: associations d'entrepreneurs, syndicats patronaux, chambres consulaires, notamment.

L'environnement intellectuel des télécommunications régionales

La production d'un discours régional sur les télécommunications fait apparaître un rôle central des cabinets d'études, au statut public ou privé, et des différents institutions chargées de l'aménagement en général. En France en particulier, le rôle de la DATAR dans le lancement et le soutien aux schémas régionaux de la communication, dans les années 1980, doit être considéré comme un des facteurs primordiaux de génèse intellectuelle du croisement décentralisation-nouvelles technologies de communication.

Dans un contexte de mise en question de son efficacité et de sa légitimité même au sein de l'appareil politico-administratif central, la DATAR a trouvé dans les télécommunications, en particulier, la matière d'un possible renouveau de ses missions, et de ses alliances avec les collectivités territoriales. En association avec des bureaux d'études tels que l'IDATE, la réalisation de ces schémas relevait, à un moment de fondation des régions comme collectivités de plein exercice, de la production d'un "prêt-à-penser" régional des nouveaux réseaux et services de télécommunication, qui s'est inscrit notamment dans les contrats de Plan Etat-Région, jusqu'à celui actuellement en cours de réalisation.

On retrouve cette même fonction intellectuelle en Italie, et dans les régions que nous avons plus particulièrement analysées. Les cabinets RESEAU et Makno-Média en constituent les figures caractéristiques. La plupart des discours publics régionaux font l'objet d'une trame commune, fondée sur une anticipation des effets de l'intégration européenne en termes de compétition et de la clarification rendue nécessaire des prestations des différentes autorités: opérateurs, Etat, collectivités territoriales.

Les différenciations politiques entre exécutifs sont d'un poids relatif dans la distinction des cabinets. Au moins au départ, les sources sont comparables, voire identiques. Elles deviennent différentes dès lors que l'on se situe à un stade plus évolué, où la place d'instituts locaux (comme la fondation Marconi à Bologne) peut relever d'arbitrages politiques spécifiques. De la même façon, la présence des instituts internes à la Région (notamment ceux 


\section{2}

chargés de la programmation et du développement économique) est un indice dacclimatation plus ou moins important du discours importé. De ce point de vue, l'Emilie-Romagne se distingue de la Lombardie par le rôle important dévolu à ASTER dans la coordination et dans la mise en oeuvre des différents aspects régionaux des télécommunications.

Une caractéristique de ces différents instituts est leur relative extériorité vis à vis des opérateurs publics et des grands industriels du secteur. Ce qui peut être a priori considéré comme un signe d'autonomie de programmation et d'indépendance intellectuelle est également à interpréter comme une exclusion vis à vis de la compétence légitime, qui reste le monopole, jalousement préservé, des appareils publics. Ceux-ci, on y reviendra, ont le plus souvent une attitude réservée à l'égard de ces initiatives, qu'elles viennent d'ailleurs de consultants localisés ou d'institutions nationales (comme la DATAR).

C'est sans doute en Espagne que l'on trouve le schéma le plus original de ce point de vue. La rédaction des Livres Blancs des télécommunications a ici fait l'objet d'une démarche contractuelle poussée entre l'opérateur Telefonica, de statut privé, et les Généralités. Qu'il s'agisse des dirigeants mêmes de l'opérateur ou de cabinets d'études liés à lui organiquement (comme Fundesco), Téléfonica semble être des trois le plus sensible à une démarche contractualisée. Celle-ci va même jusqu'à la mise à disposition de cadres dans la réalisation des projets. Cela n'implique pas la disparition des spécificités "autonomiques", au contraire. Le poids de l'Université Autonome catalane, tout comme celui de l'IMADE à Madrid, sont là pour montrer l'importance symbolique d'une traduction, autonome des discours d'ingénieurs ou d'aménageurs.

Une des raisons de cette différence entre l'Espagne, d'un côté, et l'Italie et la France de l'autre, est à rechercher dans l'histoire de chaque opérateur dans ses relations à l'Etat et au territoire. Dans le premier cas, Telefonica a, en réalité, toujours détenu un statut privé après lequel courent une partie des dirigeants des appareils similaires italien et français. De la sorte, l'actuelle montée d'aspirations régionales dans ce secteur relève de relations conventionnelles plus banalisées. En Italie et en France en revanche, cette émergence se place dans un contexte où les opérateurs parviennent à obtenir une certaine autonomie à l'égard de leur tutelle étatique traditionnelle. Elle est donc d'abord vécue 


\section{$43 ?$}

sur le mode négatif de nouvelles contraintes publiques potentielles vis à vis desquelles il s'agit de demeurer extérieur par principe. La rhétorique dominante de l'entreprise nouvelle", contre l'"administration traditionnelle" va à l'encontre d'une reconduction des structures de cette dernière, si elles paraissent inadaptées aux impératifs de développement. Cette mutation, que l'on observe aussi bien en France qu'en Italie, n'est pas pour rien dans la formalisation d'initiatives de pouvoirs locaux qui affrontent ainsi une forme de "disparition" de l'Etat dans les télécommunications territoriales.

\section{RÉSEAUX, SERVICES, PUbLICS}

Dans ces conditions d'"apprentissage intellectuel", il n'est pas étonnant de constater que les initiatives concrètes prises par les pouvoirs locaux s'orientent dans trois directions essentielles: un état des lieux régionalisé, une appropriation proche des compétences décentralisées les plus importantes, une tentative de coalition d'intérêts régionaux auprès des opérateurs. Chacune de ces entrées en matière demeure cependant marquée par l'incertitude.

\section{Quelle régionalisation des réseaux ?}

L'état des lieux constitue l'entrée commune aux pouvoirs régionaux dans les télécommunications. Il consiste à obtenir, de la part de l'opérateur public, une territorialisation des données globales de nature à orienter ensuite les initiatives politiques. Cet aspect, qui pourrait sembler anodin, soulève dans une bonne partie des Régions des enjeux considérables. Le premier relève du partage par le monopole public d'une part d'informations qu'il considère comme exclusives. A cet égard, la simple fourniture d'informations sur les flux de communication (où téléphonent les abonnés, à l'intérieur de la Région, au niveau national, à l'étranger), considérée comme une forme élémentaire de connaissance démographique et sociologique, revêt un caractère différent dès lors que le contexte européen et international est vécu comme ouvert à la dérégulation. La connaissance régionalisée des flux constitue dès lors une "information stratégique" pouvant orienter la pénétration de concurrents internationaux sur les créneaux les plus sensibles (les communications internationales en particulier). L'ouverture de filiales de British-Télécom à Milan d'abord, à Rome ensuite, 


\section{4}

tournées vers la clientèle des grandes entreprises multinationales, en est l'illustration. L'intervention, sur le territoire barcelonais, d'opérateurs européens de services pour offrir des prestations de réseau câblé appartient à la même logique. La fourniture par l'opérateur de l'état régionalisé des infrastructures et des services, parce qu'elle est aussi assimilée à une ingérence possible des pouvoirs territoriaux dans la stratégie d'une entreprise autonome, est rarement livrée facilement. Elle constitue, une fois transmise, une base de travail pour les différents acteurs, dans un contexte marqué par certaines ambiguittés.

Les Régions mettent en général l'accent sur les infrastructures. Elles constituent l'élément le plus sensible du point de vue du territoire, rapportées à des politiques de desserte empruntées à la route ou aux chemins de fer, pour lesquelles les pouvoirs locaux disposent de compétences décentralisées. Or, ce mode de lecture trahit le plus souvent une vision partielle et faussée du secteur des télécommunications. La disposition d'infrastructures n'est plus, a quelques exceptions près, le problème central de l'accès aux télécommunications. Celle des services (qui est, pour une part seulement, conditionnée par la nature des réseaux) est devenue première, au fur et à mesure que l'équipement téléphonique, dans ces régions, atteignait un degré élevé. C'est bien le sens des premières évaluations d'un programme comme STAR, centré sur le rattrapage des retards dans l'équipement des régions: la relative résorbtion des inégalités en réseau fait face à une sous-utilisation de celles-ci, notamment par les entreprises les plus directement concernées au départ. Les "retards" tendent donc à se reproduire ou à s'aggraver, non plus du fait de la desserte en réseaux mais de l'absence de socialisation locale de leurs usages. La multiplication des services, elle-même facteur de complexité et d'incertitude (sur les choix d'équipement par exemple) suppose des compétences sensiblement plus rares et coûteuses.

Cet intérêt pour les infrastructures peut également se comprendre comme l'expression d'un doute sur le bien-fondé d'une intervention dans un tel secteur.

Les réseaux relèvent en effet d'une compétence indiscutable et sans partage d'opérateurs nationaux. On peut considérer, même dans le contexte européen actuel d'introduction de la concurrence dans les grands réseaux, qu'ils demeureront leur apanage exclusif, soit par disposition réglementaire (domaines réservés où le monopole national peut continuer de s'exercer), soit par coopération 
entre acteurs au niveau communautaire. Les Régions qui ont tenté d'intervenir directement dans la création d'infrastructures ont subi presque toujours cette nationalisation des compétences, savoir-faire et enjeux. Dans le cas de la Lombardie, la stratégie un moment bienveillante de la SIP s'est rapidement montrée rétive à toute régionalisation du partenariat de réalisation du réseau "Lombardia cablata". Les projets de réseaux régionaux en France ont débouché sur des impasses comparables, dès lors qu'il s'agissait pour certaines collectivités de construire des infrastructures spécifiques. Le câble français lui-même, pourtant construit sur cette idée, a très vite renoncé à une gestion purement publique et locale des réseaux. Dans tous les cas de figure, les velleités locales affrontent simultanément les problèmes d'absence d'expertise, de relations sectorielles (notamment entre opérateurs et industriels) et interministérielles (voir le cas de RENATER) qui dépassent largement l'échelon local ou régional.

\section{Le territoire dans les stratégies d'opérateurs}

Ce problème de compétence, frontalement posé aux collectivités territoriales, s'est doublé d'une évolution, à bien des égards opposée, des opérateurs. L'autonomisation des exploitants nationaux à l'égard de l'Etat, qui ne se résume d'ailleurs pas aux prescriptions communautaires sur la séparation entre réglementation et exploitation des télécommunications, renvoie à une double dimension historique et territoriale.

Avec des temporalités légèrement différentes, les opérateurs espagnol, italiens et français ont construit cette autonomisation sur une longue période. La division formelle du territoire en Directions régionales correspondant aux découpages politico-administratifs a été, en France dès les années 1960, doublée de la création d'échelons spécifiques (les Directions opérationnelles) et de tentatives de dé-régionalisation par l'invention d'une logique de "zones" inter-régionales. Cette stratégie territoriale, tournée d'abord vers une émancipation à l'égard de la tutelle de l'administration postale, puis de celle de l'Etat central et local, était puissamment attachée au mode de pensée des ingénieurs du corps des télécommunications. Il est frappant de constater que les projets de réorganisation territoriale $y$ ont toujours coïncidé avec une ascension des institutions locales: les réformes politicoadministratives de 1964, de 1972 par exemple. 
La régionalisation des années 1980 ne fait pas exception. Au contraire de la vision, un peu formelle, d'un emboîtement hiérarchique descendant de la DGT vers les DRT puis les DOT et les ACTEL, l'organisation s'est appuyée, de manière dominante, sur les pôles opérationnels (sans référent politico-administratif obligé) plutôt que sur un pouvoir fort des Directions régionales. La fin des années 1980 voit les Directions régionales se vider d'une partie importante de leurs prérogatives (notamment celle de négocier seules le budget local avec les dirigeants centraux) et émerger une nouvelle réorganisation qui met fin, de facto, à la dernière structure territoriale coïncidant avec celle de l'Etat.

Dans le cas italien, la recomposition territoriale s'est faite plus tard, selon une logique sensiblement différente. Historiquement, la structuration territoriale de l'exploitation, au contraire de la France et de l'Espagne, a reposé sur une base régionale ou inter-régionale. Cette segmentation territoriale en opérateurs jouissant chacun d'un monopole régional, dont on ne trouve en Europe qu'un seul exemple réellement comparable au Danemark, est à rapprocher du modèle américain. Elle s'est prolongée jusque dans les années 1950, au moment où la croissance du secteur appelait, pour les dirigeants des télécommunications italiennes, une nationalisation réelle de l'exploitation. Celle-ci s'est construite selon un modèle hiérarchique à forte domination centrale, comme dans les autres pays.

En revanche, elle s'est appuyée dès le départ sur un niveau régional bien plus significatif qu'en France. Le rôle des Directions Régionales, tant dans les négociations budgétaires et stratégiques avec le milieu central qu'à l'égard des divisions provinciales et locales, s'est affirmé au cours des années 1960-1980. Les différenciations marquées entre régions italiennes, notamment du point de vue économique, ont logiquement produit

des contrastes de valeur et d'influence de ces échelons territoriaux. Une Direction Régionale comme celle de la Lombardie pouvait apparaître comme un pôle de pouvoir important dans l'organisation elle-même, ce qui a rarement été le cas en France, par exemple.

La réorganisation des structures territoriales s'est produite dans la phase finale du réaménagement institutionnel (la réunion de l'intégralité de l'exploitation dans une même holding, l'IRI). La SIP, confrontée à la montée des revendications d'utilisateurs professionnels, à l'émergence de nouveaux services et réseaux (le téléphone mobile en particulier) et à des formes de concurrence nouvelles et localisées, a remis en question la division régionale de l'exploitation. En adoptant une logique d'inspiration anglo-saxonne, 
par bassin d'usage, elle a vidé les Régions d'une partie de leurs prérogatives en les recentralisant soit directement au niveau national, soit en créant un nouvel échelon ad-hoc, inter-régional (trois zones pour le téléphone mobile, neuf pour la gestion de la clientèle "business"). L'un des effets de cette restructuration est de mettre fin à l'"intégrité territoriale" de la Région lombarde pour le domaine "business", coupée en deux entre Milan et le reste de la Lombardie.

L'Espagne est, des trois pays considérés, le seul qui inscrive le rôle des collectivités locales dans la constitution organique de l'exploitation des Télécommunications puisque les C.C.A.A. bénéficiant d'une compétence en matière de communication siègent dans un "Consejo de Assessores" organisme de régulation représentatif des acteurs du secteur. Mais il faut ajouter que leur compétence est, originellement, une compétence en matière de diffusion de signaux de télévision. La spécificité de l'organisation territoriale de l'opérateur est en grande partie liée au statut privé qui lui vient de l'histoire. Notamment, la division géographique par zones et provinces, si elle tient compte des découpages politicoadministratifs, a très tôt intègré une conception à géométrie variable. Ainsi, la hiérarchisation des provinces (circonscription de base de Telefonica), de 0 à 6 correspond elle-même à des degrés d'autonomie distincts à l'égard de la direction centrale, dans les domaines commercial, d'infrastructure ou de fourniture d'équipement. Ces directions provinciales dépendent à leur tour plus ou moins, selon leur importance, de Directions de Zones (NordEst, Nord-Ouest, Sud-Est, Sud-Ouest). Deux provinces occupent une place à part dans ce classement, qui dépend avant tout du volume d'activité concerné: Madrid et Barcelone. Pour celles-ci, la concentration de moyens est spécifique, et leur prestige dans l'organisation est attesté par le fait que leur directeur a rang de Sous-Directeur Général de l'entreprise. Elles ne dépendent pas des directions zonales, au contraire des sautres divisions provinciales. Leur influence géographique est cependant limité, pour des motivations politiques notamment. La Direction barcelonaise n'est pas compétente pour une très large partie de la Catalogne, qui dépend de la Direction de zone du Nord-Est. L'influence de la Direction madrilène, en revanche, excède les limites de la Communauté autonome.

Cette organisation territoriale est assez comparable à celle qui avait été tentée en France dans les années 1970: les délégations de zones. 


\section{8}

Circonscription inédite dans l'ordre politico-administratif, elle permet à l'entreprise Telefonica une autonomie qui a longtemps fait l'objet de résistances en France. Par ailleurs, la reprise d'un découpage provincial n'est pas totale, puisqu'il est fonction du poids économique, et de la sensibilité politique des structures décentralisées. Ce dernier aspect rapproche le cas espagnol des récentes réformes italiennes, par la division en fonction du bassin d'utilisation, et non plus par unité territoriale. La séparation Milan/reste de la Lombardie correspond à bien des égards à celle qui prévaut entre Barcelone et le reste de la Communauté autonome catalane, et pour des raisons comparables. Enfin, un même mouvement de recentralisation de la fonction commerciale est à l'oeuvre, et surtout dans le domaine des "grands clients", dont le traitement par Telefonica est désormais centralisé.

\section{Politiques de réseaux ou politiques de services}

Ces impasses ont mis en évidence un second aspect du problème: la légitimité et l'intérêt d'une intervention des pouvoirs publics locaux et régionaux dans ce domaine. Cette mise en doute n'est pas séparable en France de la contestation, plus générale, de "l'idéologie du tout-communication" des années 1980 , et en particulier de ses effets locaux. Elle est particulièrement nette dans la manière dont les exécutifs se montrent réservés à l'égard d'un domaine certes à forte valorisation symbolique, mais fort éloigné de leurs sphères de compétence, comme l'action sociale, l'urbanisme, les transports ou l'éducation.

C'est la raison pour laquelle la responsabilité de la gestion de ce domaine au sein des gouvernements locaux ne fait pas souvent l'objet d'une délégation spécifique. La lutte entre assesseurs pour un leadership sur les télécommunications en Emilie-Romagne fait figure d'exception. Dans la plupart des cas, la direction en incombe à une structure relativement marginale, par son statut et son influence, vis à vis des institutions politiques locales. Cette structure relève fréquemment de l'économie mixte, comme en EmilieRomagne, à Madrid ou en Languedoc-Roussillon.

L'un des effets majeurs de l'investissement des pouvoirs régionaux dans les télécommunications est donc moins à rechercher dans le domaine des infrastructures que dans celui des services.

En France en particulier, à une phase d'enthousiasme pour les artères de communication a succédé une polarisation sur la 
prestation de services, aux entreprises et au public en général. Cet intérêt nouveau, outre qu'il est lié aux impasses d'une réelle politique de réseaux régionaux, consacre un recentrage sur les compétences décentralisées, ou en voie de l'être à terme. C'est le cas des services en matière de recherche, avec RENATER, ou encore du soutien à des services spécifiques d'enseignement (formation à distance, cours téléphonés...).

En Italie, le développement des services régionaux de télécommunication passe par les stratégies d'innovation propres à chaque segment de l'appareil régional. L'Emilie-Romagne se distingue, de ce point de vue, par le nombre des initiatives sectorielles, souvent liées à la télématique, que l'exécutif régional a développé par l'intermédiaire de ses institutions de recherche et de programmation économique. L'institution régionale joue dans ce secteur un rôle important d'encadrement du développement, des petites et moyennes entreprises notamment, qui avait fait sa spécificité idéologique: l'alliance de la domination politique communiste et des intérêts des petits entrepreneurs. Nombre des initiatives concernent également la modernisation des services publics locaux, pour lesquels le soutien des financements européens de recherche-développement est le plus important. La différence est nette avec les initiatives régionales lombardes, essentiellement produites, sur les infrastructures, par les intérêts des grands acteurs nationaux du secteur, et faibles voire inexistantes en matière de service.

\section{L'approche fédérative des collectivités territoriales}

L'émergence des pouvoirs régionaux dans les télécommunications, confrontée à une résistance farouche des opérateurs nationaux, a également pris, au tournant des années 1990, une dimension fédérative. Les tentatives des collectivités territoriales d'établir, face à l'opérateur, une forme de coalition d'intérêts sont toutefois très différentes d'un pays à l'autre. Ces différences recoupent d'ailleurs logiquement celles que nous avons décrites à propos du rôle et de l'intensité de la régionalisation.

En France, d'un côté, cette rationalisation des relations collectivités locales-France-Télécom a été directement impulsée par le milieu décisionnel central, autour de la réforme du statut de l'opérateur. La Mission, le Rapport, puis la Délégation aux "Télécommunications 
dans la Ville" font figure de réaffirmation des missions de service public d'un opérateur en voie d'autonomisation et dont le statut d'entreprise vient d'être consacré. La Délégation vient apporter en outre une réponse aux constats de carence relationnelle de FranceTélécom au niveau local, dréssés par le Rapport Schreiner. Elle institutionnalise une fonction de filtre des revendications des pouvoirs locaux à l'égard de l'opérateur public. Il n'est pas anodin, dans cette perspective, que la Délégation soit rattachée directement à France-Télécom, et non pas au Ministère ou encore au Parlement. L'institutionnalisation italienne de cette fonction de médiation Régions-opérateurs publics est rigoureusement inverse. C'est d'une initiative régionale (en Emilie-Romagne) que naît pour la première fois l'idée d'une forme de conférence des Régions ad-hoc, baptisée ORESTE (Observatoire Régional Sur les TElécommunications), ayant pour fonction d'inventorier les initiatives régionales, en matière de services et réseaux, et de constituer le pôle autour duquel opérateur national, prestataires privés, Commission Européenne et Régions entreraient en relation. Cette première démarche collective des pouvoirs régionaux se donne également pour mission l'échange d'expériences, l'assistance et plus généralement la diffusion de l'imprécise, bien que toujours soulignée, "culture des télécommunications".

Cette "culture des télécommunications" fait apparaître la différence entre secteurs que certains considèrent comme inéluctablement convergents: l'audiovisuel et les télécommunications. Le premier relève d'une problématique plus ancienne, de logiques d'acteurs très différents et de centres d'intérêt régional opposés. Les Régions italiennes et espagnoles ont, beaucoup plus tôt que les françaises, fait du soutien à la diffusion télévisuelle régionale un véritable axe de politique publique. Les chaînes régionales ou autonomiques relèvent d'une problématique d'identité culturelle, de programmation de contenus, de citoyenneté régionale. Les télécommunications sont à l'inverse vécues à partir du développement économique, d'une catégorie beaucoup plus spécifique de l'espace public régional, et sont d'abord envisagées comme infrastructures. Elles font appel à des professions spécifiques, dont l'expertise est infiniment plus concentrée et pour lesquelles les pouvoirs locaux sont en quête d'une légitimité incertaine. 


\section{POlitiQues RÉgionales, SERVICE PUblic ET EUROPE}

Dans le contexte de libéralisation du secteur, la situation de pays ou de régions moins avancés est spécifique. Les collectivités interviennent dans un secteur moins développé, bien que soumis aux mêmes principes d'évolution que les autres Etats-membres de la CEE. Leurs initiatives se situent alors dans le prolongement d'un service réputé rendu dans des conditions insatisfaisantes par l'opérateur national: c'est le cas des dispositifs concernant la prise en charge d'un surcoût territorial d'équipement lié à une localisation éloignée, ou à des ressources financières insuffisantes. Péréquation territoriale et égalisation des usages en incarnent la philosophie. Le financement demandé aux Régions françaises pour le développement des "plaques régionales" du Réseau RENATER s'apparente à une participation des pouvoirs régionaux au service public lui-même.

Au delà des infrastructures, leur intervention dans la diffusion des services prend différentes formes: des prestations de formation à l'usage des nouveaux services, le financement de services euxmêmes (télématiques notamment), l'intégration des innovations technologiques dans la prestation de services publics locaux (transports, secteur sanitaire et social, éducation, informations publiques et administratives...). Cette intervention ne peut plus être considérée, par les opérateurs, comme une simple ingérence de l'Etat, au niveau local, dans la politique d'une entreprise. Cette image des échelons locaux continue d'être dominante, mais elle est travaillée de l'intérieur par de nouvelles logiques relationnelles, fondées sur la contractualisation notamment, dans le contexte d'ouverture à la concurrence de certains domaines d'activité. Les interventions régionales, encore timides, sont liées à la modernisation des télécommunications elles-mêmes, la complexification des services, de leurs usages et de leur régulation locale. C'est essentiellement dans cette logique que la régionalisation est considérée comme une forme de redéploiement du service public: autour des services, de leur diffusion auprès d'acteurs économiques liés aux politiques publiques régionales, comme les PME. 
Mais ces politiques régionales représentent aussi une potentielle remise en cause du service public. Derrière les formes variables d'action collective des régions face aux appareils nationaux de télécom, la promotion des régions comme acteur potentiel se situe dans le contexte de mise en concurrence des espaces, a l'échelle européenne. Les revendications tendant à une nouvelle appréciation des rapports offre de télécommunication - structures de la demande économique localisée se situent aux antipodes des principes de péréquation, fondateurs du service public au sens large. C'est toute l'ambiguïté d'un local-corporatisme, notamment dans le cas italien, où ces initiatives sont directement confrontées à de forts déséquilibres inter-régionaux. Ce n'est pas un hasard si ces politiques sont menées d'abord, et toujours essentiellement, par des régions riches et développées (Lombardie, Catalogne...), qui jouent ce rôle de mise en question du monopole et articulent un discours de niveau et de contenu "européens" face à leurs Etats respectifs 3.

Le deuxième enjeu réside précisément dans le rapport à l'Europe de ces politiques régionales. Il y a au moins deux manières d'aborder ce rapport.

Le rapport de l'Europe aux régions est d'abord "stratégique", en ce que le soutien remarquable, de la part de la Commission, de ce type de politique sert objectivement ses intérêts institutionnels, en affaiblissant le niveau de souveraineté et de contrôle des Etats, notamment en ce qui concerne certains aspects liés aux politiques industrielles. Les glissements progressifs dans l'interprétation du Traité de Rome sur les monopoles, les tentatives de la Direction à la Concurrence d'imposer un nouvel ordre économique et juridique participent de cette évolution. Elles reposent sur une vision péjorative de l'Etat en termes de coût, ou de surcoût. Implicitement, la remise en question du service public de communication est l'un des objets de cette solidarités inter-institutionnelles. Elle a cependant fort à faire avec le maintien d'une puissante influence des appareils publics et de leurs représentants dans les instances communautaires.

La présence, notamment au titre des Fonds structurels, d'une politique de la Commission (DG XIII-DG XVI) en faveur des régions

\footnotetext{
3.C'est notamment le cas du "Quadrige" des Régions du Bade-Wurtemberg, de la Catalogne, de la Lombardie et de Rhone-Alpes, qui a récemment rédigé, tout à fait dans ce sens, un "Memorandum sur les télécommunications".
} 
les moins favorisées renvoie à un autre niveau. Le programme STAR, puis TELEMATIQUE, en fait partie (Pascal 1991). Le paradoxe est que cette politique n'est pas régionale, au sens où la Commission n'entre pas en relation directe avec les régions, mais que ce programme est "filtré" par les Etats, avec des conditions variables de coopération des instances locales. La politique européenne est ici assez directement liée à un des principes de base du service public: l'égalisation des conditions d'accès aux infrastructures et services, en même temps qu'elle est contrainte par une appréciation à dominante nationale de la subsidiarité. On peut s'interroger, à titre prospectif, ce que deviendra l'application de ce principe à des pays ou des régions plus faiblement encadrés. D'ores et déjà, la participation directe d'experts de la Commission à la mise en place et au développement de nouveaux réseaux et services heurte le schéma. Elle peut être à l'origine d'une conception du service public européen des télécommunications originale, tant au niveau des acteurs que de celui des échelles d'application des principes. Une étude de ces politiques de la Communauté dans les régions désignées comme appelant de puissantes interventions "de cohésion" (dans les régions "périphériques" espagnoles, dans le Mezzogiorno, en Grèce et au Portugal) permettrait d'en dégager le contenu.

Il est évident que ces deux modes d'articulation ne sont pas harmonieux, et qu'ils se situent au coeur des conflits qui traversent la construction européenne, entre politiques publiques, libéralisation et solidarités territoriales. Le cas des télécommunications en constitue un des révélateurs. 
ALLIES P.: "L'intégration des collectivités territoriales françaises dans le système des politiques publiques communautaires", dans les Actes du colloque de l'Institut de la Décentralisation: "La décentralisation française et l'Europe" Strasbourg, Novembre 1992 BEGAG A., CLAISSE G.: "Téléinformatique et localisation des entreprises" France Télécom n77, Mai 1991

BIANCHI A., RICHERI G. (1991): "Une politique régionale pour les télécommunications: le cas de l'Italie" in "Quelles politiques régionales pour les télécommunications ?" Cabinet de l'Aménagement du Territoire de la Région Wallonne, Le Grand Hornu, Octobre 1991

BRIOLE A., LAURAIRE R.: "Les réseaux de télécommunication d'intérêt local" Revue QUADERNI N¹3-14, 1991

CAMMELLI (1990): "Regioni e rappresentanza degli interessi: il caso italiano" Stato e Mercato $n^{\circ} 29$, Il Mulino Bologne

CARPENTIER M., FARNOUX-TOPORKOFF S., GARRIC C. (1991): "Les

télécommunications en liberté surveillée" Lavoisier, Paris

COHEN E. (1991): "Le colbertisme high tech" Hachette Paris

DE LA TORRE L.: "le Département saisi par la communication"

Quaderni $n^{\circ} 13-14$, Paris 1991

DOUTRIAUX Y.: "La politique régionale de la CEE" Que Sais-je ?, PUF 1991

DREVET J.F.: "La politique régionale communautaire et la France" dans les Actes du colloque de l'Institut de la Décentralisation: "La décentralisation française et l'Europe" Strasbourg, Novembre 1992 DUCASTEL F., CHAMBAT P., MUSSO P.: "L'ordre communicationnel. Les nouvelles technologies de la communication: enjeux et stratégies" CNET-Documentation Française 1989

ENSPTT: "Management des entreprises de réseau" Actes du colloque 10-11 janvier 1992

Revue Esprit : "La Communauté Européenne et les chocs de l'Histoire" $\mathrm{n}^{\circ} 11$, Paris Novembre 1991

EVENO: "Territoire régional et réseaux de communication électronique. L'évolution des enjeux" GRESOC-Université de Toulouse-Le Mirail 1992

GENSOLLEN M.: "Les réformes institutionnelles et réglementaires des télécommunications en 1990: le service public face à l'extension de la concurrence" Communication \& Stratégies $n^{\circ} 3$, IDATE Montpellier 1991

GREENWOOD J., GROTE J., RONIT K.: "Organized interests and the European Community" Sage Publications, Londres 1993 HUSSON J.P.-PEREZ Y. (1992): "L'Europe industrielle naîtra-t-elle dans les régions ?" Le Monde diplomatique, Mars 1992 
LASSERRE B.: "l'Europe des télécommunications: les options de la CEE et la position de la France" Revue Les Annales des Mines, avril 1991 MURRAY R. "Local space: Europe and the new regionalism" CLES, Manchester 1991

NEGRIER E., RABATE F.: "Le développement régional des services de communication" in "Quelles politiques régionales pour les télécommunications ?" Cabinet de l'Aménagement du Territoire de la Région Wallonne, Le Grand Hornu, Octobre 1991 PASCAL O. (1991): "Les télécommunications avancées au service des régions périphériques" XIII Magazine ${ }^{\circ} 2$, CCE-DG XIII, Juillet PELASSY D.: "Qui gouverne en Europe" Fayard, Paris 1992 RITAINE E.: "Changement social et pratiques développementalistes en Languedoc-Roussillon" Rapport pour le MRT, CERVL Bordeaux 1991

SCHMITTER P.C.: "The European Community as an emergent and novel form of political domination" Séminaire de l'OPPES (Observatoire des Politiques Publiques en Europe du Sud), Montpellier Avril 1991

SCHREINER B.: "Les télécommunications dans la Ville" Rapport au Ministre des PTE 1990

SIMON J.P.: "L'Esprit des règles. Réseaux et réglementation aux Etats-Unis" L'Harmattan 1991

STEVERS E.: "Administative reform at the European Commission and the development of a telecommunications policy" Actes du Congrès de l'European Consortium of Political Research, Bochum Avril 1990 STEVERS E.: "Telecommunication regulation in the European Community. The Commission of the European Communities as regulatory actor" Working Paper $n^{\circ}$ 89/421 Institut Universitaire Européen, Florence 1990

TORNOS-MAS J.: "Regioni e rapresentanza degli interessi: il caso catalano" Stato e Mercato ${ }^{\circ} 29$ précité

VALADOU P.: Quel avenir pour les collectivités locales françaises autres que les Régions ?" dans les Actes du colloque de l'Institut de la Décentralisation: "La décentralisation française et l'Europe" Strasbourg, Novembre 1992

A.VALLEEE: "Quels opérateurs, pour quels réseaux et quels services ?" Séminaire de l'ENPC, Paris 1991

VANDELLI L. (1991): "Pouvoirs locaux" Economica, Paris 